\title{
Lupeol and pristimerin do not inhibit activation of the human
}

\section{sperm CatSper $\mathrm{Ca}(2+)$-channel [version 1; peer review: 3}

\section{approved with reservations]}

\author{
Anders Rehfeld (D1) 1-3, Christian Marcus Pedersen (Di)4 \\ ${ }^{1}$ Department of Drug Design and Pharmacology, Faculty of Health Sciences, University of Copenhagen, Copenhagen, 2200 \\ Copenhagen N, Denmark \\ ${ }^{2}$ Department of Biomedical Sciences, Faculty of Health Sciences, University of Copenhagen, Copenhagen, 2200 Copenhagen N, \\ Denmark \\ ${ }^{3}$ Department of Growth and Reproduction, University of Copenhagen, Rigshospitalet, Copenhagen, 2100 Copenhagen E, Denmark \\ ${ }^{4}$ Department of Chemistry, University of Copenhagen, Copenhagen, 2200 Copenhagen N, Denmark
}

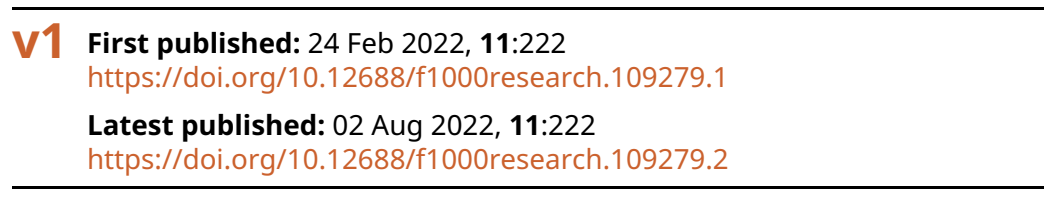

\section{Abstract}

Opposing findings have been published on the regulation of the sperm-specific $\mathrm{Ca}^{2+}$ channel CatSper (cation channel of sperm) in human sperm cells by the plant triterpenoids lupeol and pristimerin. While the original study on this topic found these triterpenoids to act as potent inhibitors of human CatSper, subsequent studies have failed to replicate such an inhibitory effect. It has been suggested that these issues could in part be due to purity issues and/or batch variation between the plant-derived extracts of lupeol and pristimerin obtained for the studies. The aim of this study was to elucidate this controversy by investigating the batches of lupeol and pristimerin used in our previous study with state-of-the-art ${ }^{1} \mathrm{H}-,{ }^{13} \mathrm{C}$ - and $2 \mathrm{D}$-nuclear magnetic resonance (NMR) methods to reveal potential purity and/or batch variation issues. When comparing the NMR-spectra obtained from ${ }^{1} \mathrm{H}$ NMR and ${ }^{13} \mathrm{C}-\mathrm{NMR}$ with previously published NMR-spectra for lupeol and pristimerin, we could confirm that both the lupeol and pristimerin batch were $\geq 95 \%$ pure. These results confirm the validity of the findings in our previous study for lupeol and pristimerin, showing that lupeol and pristimerin do not inhibit activation of CatSper in human sperm. In conclusion, using ${ }^{1} \mathrm{H}-,{ }^{13} \mathrm{C}$ - and $2 \mathrm{D}$-NMR methods, we confirm that the lupeol and pristimerin batches used in our previous study were $\geq 95 \%$ pure and thereby fail to identify any purity issues and/or batch variation that could explain the observed inability of lupeol and pristimerin to inhibit activation of CatSper in human sperm.

\section{Keywords}

Fertility, CatSper, Male reproduction, Lupeol, Pristimerin, Sperm function

\section{Open Peer Review}

$\begin{array}{rrr}\text { Approval Status } \\ 1 & 2 & 3\end{array}$

version 2

(revision)

02 Aug 2022

version 1

$24 \mathrm{Feb} 2022$

$\begin{array}{cc}\checkmark & \\ \text { view } & \text { view } \\ ? & ? \\ \text { view } & \text { view }\end{array}$

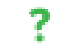

view

\section{João Ramalho-Santos (D), University of}

Coimbra, Coimbra, Portugal

University of Coimbra, Coimbra, Portugal

University of Coimbra, Coimbra, Portugal

2. Tao Luo ID, Nanchang University,

Nanchang, China

3. Huafeng Wang (iD), Yale School of Medicine,

New Haven, USA

Any reports and responses or comments on the article can be found at the end of the article. 
Corresponding authors: Anders Rehfeld (rehfeld@sund.ku.dk), Christian Marcus Pedersen (cmp@chem.ku.dk)

Author roles: Rehfeld A: Conceptualization, Funding Acquisition, Project Administration, Writing - Original Draft Preparation, Writing Review \& Editing; Marcus Pedersen C: Data Curation, Methodology, Visualization, Writing - Review \& Editing

Competing interests: Anders Rehfeld holds a patent P2493DK00 "ZAFIRLUKAST DERIVATIVES FOR USE AS CONTRACEPTIVE AGENTS".

Grant information: AR is funded by a BRIDGE - Translational Excellence Programme grant funded by the Novo Nordisk Foundation, grant agreement number: NNF18SA0034956.

The funders had no role in study design, data collection and analysis, decision to publish, or preparation of the manuscript.

Copyright: $\odot 2022$ Rehfeld A and Marcus Pedersen C. This is an open access article distributed under the terms of the Creative Commons Attribution License, which permits unrestricted use, distribution, and reproduction in any medium, provided the original work is properly cited.

How to cite this article: Rehfeld A and Marcus Pedersen C. Lupeol and pristimerin do not inhibit activation of the human sperm CatSper $\mathrm{Ca}(2+)$-channel [version 1; peer review: 3 approved with reservations] F1000Research 2022, 11:222

https://doi.org/10.12688/f1000research.109279.1

First published: 24 Feb 2022, 11:222 https://doi.org/10.12688/f1000research.109279.1 
The putative inhibitory action of the two plant triterpenoids lupeol and pristimerin on the activation of the human sperm CatSper $\mathrm{Ca}(2+)$-channel has recently been debated in the scientific literature. The original study on this subject (Mannowetz et al., 2017) indicated that these triterpenoids act as very potent and efficacious inhibitors of progesterone-activated CatSper-currents in human sperm cells with $\mathrm{IC}_{50}$-values in the lower nM range, and a follow-up study by the same research group confirmed the inhibitory action of pristimerin on progesterone-induced $\mathrm{Ca}(2+)$-influxes via CatSper through measurements in the principal piece of the flagellum in single human sperm cells (Mannowetz et al., 2018).

In contrast to these findings, two studies from independent research groups failed entirely to replicate any inhibitory action for neither lupeol nor pristimerin on progesterone-induced $\mathrm{Ca}(2+)$-influxes through CatSper in populations of human sperm cells (Brenker et al., 2018; Rehfeld, 2020) and progesterone-activated CatSper-currents in single human sperm cells (Brenker et al., 2018), even when exposing the sperm cells to lupeol and pristimerin at much higher $\mu \mathrm{M}$ concentrations.

The complete failure of these studies to replicate the findings from (Mannowetz et al., 2017; Mannowetz et al., 2018) is highly concerning since a patent has been filed (Lishko \& Mannowetz, 2018) and a company (YourChoice Therapeutics, CA, US) has been formed based on the original discovery by (Mannowetz et al., 2017; Mannowetz et al., 2018) that lupeol and pristimerin act as potent inhibitors of human CatSper and could thus potentially be used as novel male and female contraceptives.

Since the publication of the most recent study on this matter (Rehfeld, 2020), the corresponding author was contacted by researchers who questioned the validity of the results presented in the study for lupeol and pristimerin, i.e., the inability to reproduce the inhibitory action of these triterpenoids on human CatSper, and suggested that the failure to identify such an inhibitory effect on human CatSper could be due to purity issues and/or batch variation between the plant-derived extracts of lupeol and pristimerin obtained for the study from Cayman Chemicals (MI, USA).

Although Cayman Chemicals stated that the lupeol and pristimerin batches were delivered with a purity of $\geq 98 \%$, we fully agreed with these researchers that it would be good scientific conduct and of general interest of the field of human sperm physiology to examine the two stocks solutions used in (Rehfeld, 2020), i.e., a $5 \mathrm{mM}$ pristimerin dimethylsulfoxid (DMSO) stock and a $1 \mathrm{mM}$ lupeol ethanol stock, using state-of-the-art ${ }^{1} \mathrm{H}-,{ }^{13} \mathrm{C}$ - and $2 \mathrm{D}$-nuclear magnetic resonance (NMR) methods (Bruker $500 \mathrm{MHz}$ Ultrashield Plus equipped with a CryoProbe, Bruker, Germany) to reveal potential purity and/or batch variation issues in these stocks.
To prepare the stocks for the NMR-measurements, we first evaporated the ethanol from the lupeol stock, removed the DMSO from the pristimerin stock using an evaporation system (V-10 evaporator, Biotage, Sweden), and exchanged the solvent for both triterpenoids to deuterated chloroform $\left(\mathrm{CDCl}_{3}\right)$. The raw NMR data can be found as Underling data (Rehfeld, 2022a).When comparing the NMR-spectra obtained on the two stocks from ${ }^{1} \mathrm{H}-\mathrm{NMR}$ and especially ${ }^{13} \mathrm{C}-\mathrm{NMR}$ (see Extended data (Rehfeld, 2022b)) with previously published NMR-spectra for lupeol and pristimerin (Espindola et al., 2018; Shwe et al., 2019), we could confirm that Cayman Chemicals had indeed provided us with batches containing lupeol and pristimerin, respectively. Furthermore, the NMR-data showed that both lupeol and pristimerin were $\geq 95 \%$ pure (Extended data (Rehfeld, 2022b)), despite the prolonged storage at $-20{ }^{\circ} \mathrm{C}$ since conducting the experiments for (Rehfeld, 2020).

Taken together, the results provided here confirms the validity of the findings in our previous study for lupeol and pristimerin (Rehfeld, 2020), i.e., that the two plant triterpenoids lupeol and pristimerin do not inhibit activation of CatSper in human sperm. The findings in (Rehfeld, 2020) are therefore still in line with the observations by (Brenker et al., 2018) and still contradicting the putative inhibitory action of lupeol and pristimerin on human CatSper described in (Mannowetz et al., 2017; Mannowetz et al., 2018).

In conclusion, using state-of-the-art ${ }^{1} \mathrm{H}-,{ }^{13} \mathrm{C}-$ and 2D-NMR methods, we confirm here that the lupeol and pristimerin stocks used in (Rehfeld, 2020) were $\geq 95 \%$ pure and thereby fail to identify any purity issues and/or batch variation that could explain the observed inability of these triterpenoids to inhibit activation of CatSper in human sperm.

\section{Data availability}

Underlying data

Figshare. Raw ${ }^{1} \mathrm{H}-,{ }^{13} \mathrm{C}$ - and 2D-NMR data for lupeol and pristimerin in MestReNova (Mnova) format. https://doi.org/10.6084/ m9.figshare.19181087.v1 (Rehfeld, 2022a).

Data are available under the terms of the Creative Commons Zero "No rights reserved" data waiver (CCO 1.0 Public domain dedication).

Raw ${ }^{1} \mathrm{H}-$, ${ }^{13} \mathrm{C}$ - and $2 \mathrm{D}-\mathrm{NMR}$ data for lupeol and pristimerin in MestReNova (Mnova) format are also available at the BMRbig repository, part of the Biological Magnetic Resonance Data Bank (BMRB), with ID: BMRbig35, https://bmrbig.org/ released/bmrbig35.

\section{Extended data}

Figshare: Supplementary file 1. https://doi.org/10.6084/m9. figshare.19134488.v1 (Rehfeld, 2022b).

Data are available under the terms of the Creative Commons Attribution 4.0 International license (CC-BY 4.0). 
Brenker C, Schiffer C, Wagner IV, et al.: Action of steroids and plant triterpenoids on CatSper $\mathrm{Ca}^{2+}$ channels in human sperm. Proc Natl Acad SCi USA. 2018; 115(3): E344-E346.

PubMed Abstract | Publisher Full Text | Free Full Text

Espindola LS, Dusi RG, Demarque DP, et al.: Cytotoxic Triterpenes from Salacio crassifolia and Metabolite Profiling of Celastraceae Species. Molecules. 2018; 23(6): 1494

PubMed Abstract | Publisher Full Text | Free Full Text

Lishko PV, Mannowetz N: Contraceptive use of triterpenoids. World Intellectual Property Organization. 2018.

Reference Source

Mannowetz N, Miller MR, Lishko PV: Regulation of the sperm calcium channel CatSper by endogenous steroids and plant triterpenoids. Proc Nat Acad Sci U S A. 2017; 114(22): 5743-5748.

PubMed Abstract | Publisher Full Text | Free Full Text
Mannowetz N, Mundt N, Lishko PV: Reply to Brenker et al.: The plant triterpenoid pristimerin inhibits calcium influx into human spermatozoa via CatSper. Proc Natl Acad Sci U S A. 2018; 115(3): E347-E348.

PubMed Abstract | Publisher Full Text | Free Full Text

Rehfeld A: Revisiting the action of steroids and triterpenoids on the human sperm Ca ${ }^{2+}$ channel CatSper. Mol Hum Reprod. 2020; 26(11): 816-824.

PubMed Abstract | Publisher Full Text

Rehfeld A: NMR dataset of Lupeol and Pristimerin. figshare. Dataset. 2022a. http://www.doi.org/10.6084/m9.figshare.19181087.v1

Rehfeld A: Supplementary file 1.docx. figshare. Dataset. 2022b.

http://www.doi.org/10.6084/m9.figshare.19134488.v1

Shwe HH, Win KK, Moe TT, et al.: Isolation and Structural Characterization of Lupeol from the Stem Bark of Diospyros ehretioides Wall. IEEE-SEM. 2019; 7(8): 140-144.

Reference Source 


\section{Open Peer Review}

\section{Current Peer Review Status: ? ? ?}

\section{Version 1}

Reviewer Report 04 May 2022

https://doi.org/10.5256/f1000research.120762.r129827

(C) 2022 Wang H. This is an open access peer review report distributed under the terms of the Creative Commons Attribution License, which permits unrestricted use, distribution, and reproduction in any medium, provided the original work is properly cited.

\section{Huafeng Wang}

Department of Cellular and Molecular Physiology, Yale School of Medicine, New Haven, CT, USA

The effects of lupeol and pristimerin on human CatSper have aroused a controversy since two PNAS papers were published from different groups (Mannowetz et al., 2017 and Brenker et al., 2018). This manuscript was aiming to uncover a potential possibility for a better explanation by examining the purity of lupeol and pristimerin, together with their previous work (Molecular Human Reproduction, 2020). It is believed the data in this manuscript was solid and convincing. However, is the lupeol and pristimerin tested in this manuscript from same batch with the other two groups? If not, it would be great to test those from the two groups individually, even though the purity was not mentioned in this controversy (Mannowetz et al., 2018 and Brenker et al., 2018).

Is the rationale for commenting on the previous publication clearly described?

Yes

Are any opinions stated well-argued, clear and cogent? Partly

Are arguments sufficiently supported by evidence from the published literature or by new data and results?

Partly

Is the conclusion balanced and justified on the basis of the presented arguments? Partly

Competing Interests: No competing interests were disclosed.

Reviewer Expertise: sperm, CatSper, ion channel, electrophysiology

I confirm that I have read this submission and believe that I have an appropriate level of expertise to confirm that it is of an acceptable scientific standard, however I have 


\section{significant reservations, as outlined above.}

Author Response 05 Jul 2022

\section{Anders Rehfeld}

We have now contacted these groups to offer them NMR-analyses of their lupeol and pristimerin batches.

Competing Interests: No competing interests were disclosed.

Reviewer Report 13 April 2022

https://doi.org/10.5256/f1000research.120762.r129824

(C) 2022 Luo T. This is an open access peer review report distributed under the terms of the Creative Commons Attribution License, which permits unrestricted use, distribution, and reproduction in any medium, provided the original work is properly cited.

\section{Tao Luo}

Institute of Life Science, School of Life Sciences, Nanchang University, Nanchang, China

Different research groups showed contradictory results on whether plant triterpenoids, lupeol and pristimerin, inhibit the activation of CatSper. It has been suggested that these issues could in part be due to purity issues and/or batch variation between the plant-derived extracts of lupeol and pristimerin obtained for the studies.

This manuscript aimed to elucidate this controversy by investigating the batches of lupeol and pristimerin used in their previous study using state-of-the-art 1H-, 13C- and 2D-nuclear magnetic resonance (NMR) methods. The authors confirm that the lupeol and pristimerin batches used in their previous study were $\geq 95 \%$ pure. Therefore, they concluded that the purity issues and/or batch variation could not explain the inability of lupeol and pristimerin to inhibit activation of CatSper in human sperm found in their previous study. The opinions stated are well-argued, clear and cogent. The arguments were sufficiently supported by evidence from the new data.

However, the manuscript did not discuss or respond to the other two important issues argued by Mannowetz et al. 2018:

1. An electrical driving force of $20 \mathrm{mV}$ generated from a holding potential of -80 to $-100 \mathrm{mV}$, as shown by Brenker et al. 2018, is neither enough to reliably assess inward CatSper currents nor to estimate "fold current increase".

2. If it is of interest to study CatSper-mediated calcium influx into spermatozoa, individual principle pieces (PPs) as regions of interest must be analyzed.

If these issues are addressed, the controversy may be better elucidated.

\section{Is the rationale for commenting on the previous publication clearly described?}


Yes

Are any opinions stated well-argued, clear and cogent?

Yes

Are arguments sufficiently supported by evidence from the published literature or by new data and results?

Yes

Is the conclusion balanced and justified on the basis of the presented arguments? Yes

Competing Interests: No competing interests were disclosed.

Reviewer Expertise: Male infertility

I confirm that I have read this submission and believe that I have an appropriate level of expertise to confirm that it is of an acceptable scientific standard, however I have significant reservations, as outlined above.

Author Response 05 Jul 2022

Anders Rehfeld

We have now addressed these two issues in the discussion of the updated manuscript.

Competing Interests: No competing interests were disclosed.

Reviewer Report 02 March 2022

https://doi.org/10.5256/f1000research.120762.r125363

(C) 2022 Ramalho-Santos J. This is an open access peer review report distributed under the terms of the Creative Commons Attribution License, which permits unrestricted use, distribution, and reproduction in any medium, provided the original work is properly cited.

João Ramalho-Santos

${ }^{1}$ CNC-Center for Neuroscience and Cell Biology, University of Coimbra, Coimbra, Portugal

2 DCV-Department of Life Sciences, University of Coimbra, Coimbra, Portugal

3 CIBB-Center for Innovative Biomedicine and Biotechnology, University of Coimbra, Coimbra, Portugal

My comments have to do with the fact that, while the topic is very worthwhile, this is not exactly (in my view) a straightforward Correspondence. The steps are as follows:

1. Originally a paper is published in Proceedings of the National Academy of Sciences of the United States of America (PNAS) in 2017 showing that Lupeol and pristimerin strongly 
inhibits the CatSper channel, the implication being that they could be used in male contraception, and apparently a company was formed with that in mind.

2. In 2018 (also in PNAS) other authors question this data suggesting that there are, in fact, no effects.

3. The original 2017 authors contest this, stand by their results and suggest that their data is valid and that the negative results are based on monitoring different things. Sample purity is not mentioned in this exchange.

4. It should be noted that none of the authors of this correspondence were involved in this controversy, the first author did work with the team that published the 2018 paper, and confirmed the absence of results with CatSper not being inhibited by Lupeol and pristimerin in a 2020 Molecular Human Reproduction (MHR) paper.

In this correspondence the authors perform NMR spectra to show that Lupeol and pristimerin are as pure as possible, and that the absence of inhibition of CatSper is therefore a true result. One authors is an expert on sperm (and CatSper in particular), the other on chemical structures, so I have no issues with the data here at all. However, I doubt that this will in any meaningful way solve the contradiction. Using other batches of Lupeol and pristimerin might be a possibility, or at least framing the discussion a bit more thoroughly and discussing/updating the issues raised in the 2018 exchange, that, as stated, never mention sample purity. But I do respect the authors not wanting to do this, as they were not part of those papers.

Is the rationale for commenting on the previous publication clearly described? Partly

Are any opinions stated well-argued, clear and cogent?

Yes

Are arguments sufficiently supported by evidence from the published literature or by new data and results?

Partly

Is the conclusion balanced and justified on the basis of the presented arguments? Partly

Competing Interests: No competing interests were disclosed.

Reviewer Expertise: Sperm physiology, male and female infertility, reproductive and stem cell biology.

I confirm that I have read this submission and believe that I have an appropriate level of expertise to confirm that it is of an acceptable scientific standard, however I have significant reservations, as outlined above.

Author Response 03 Mar 2022 


\section{Anders Rehfeld}

I fully agree with the reviewer in his comment that this is not a straightforward Correspondence. I would have preferred to be able to publish all my data on this issue as a Letter to the original PNAS paper, but unfortunately, there was a 6-month limit to submit such Letters to articles in PNAS and I did not have any data ready within that timeframe. My way into writing this Correspondence was this:

1. I read the original 2017 PNAS paper and was very excited about the novel CatSperinhibitor compounds Lupeol and Pristimerin, which seemed to be highly potent and efficacious compared to other known CatSper-inhibitors. I therefore ordered these compounds to use them in my own studies as CatSper-inhibitors.

2. I received Lupeol and Pristimerin, diluted them according to the recommendation of the vendor and started testing them in my own experimental setup. However, I could not identify any inhibitory effects of the compounds on CatSper in human sperm cells.

3. Puzzled, I contacted the authors of the original 2017 PNAS paper to discuss what I might have done wrong. The authors of the original 2017 PNAS paper suggested me to test Lupeol and Pristimerin prepared at different stock concentrations in different solvents, with or without sonication, as well as from different vendors. However, in the end I could still not observe any inhibition of human CatSper in my assay.

4. At this point I decided to re-examine both the two triterpenoids, Lupeol and Pristimerin, and the steroids investigated in the original 2017 PNAS paper using my own experimental setup. This collected work is what I published in the 2020 Molecular Human Reproduction paper.

5. After publishing this 2020 Molecular Human Reproduction paper, I was contacted per e-mail by researchers who questioned the validity of the results presented in the study for Lupeol and Pristimerin, and suggested that my failure to identify inhibitory effects on human CatSper could be due to purity issues and/or batch variation between the plant-derived extracts of Lupeol and Pristimerin obtained for the study. As the reviewer states, this purity issue was not mentioned in the 2018 PNAS exchange.

6. I had to agree with the researchers that this could of course be true and something that I should have investigated before submitting the 2020 Molecular Human Reproduction paper. I thus contacted, Christian Marcus Pedersen, a chemical scientist from the chemical department at the University of Copenhagen, and asked him to perform nuclear magnetic resonance analyses of the Lupeol and Pristimerin stocks I used for the 2020 Molecular Human Reproduction study.

7. Christian Marcus Pedersen performed nuclear magnetic resonance analyses of my Lupeol and Pristimerin batches and thankfully both compounds were present at high purity, even despite the prolonged storage at $-20^{\circ} \mathrm{C}$ since conducting the 
experiments for the 2020 Molecular Human Reproduction paper.

8. At this point I simply wanted to share these additional important data, which I thought were highly relevant for the discussion of the putative inhibitory effects of Lupeol and Pristimerin on human CatSper. Unfortunately, it was not possible for me to simply add these nuclear magnetic resonance data to the 2020 Molecular Human Reproduction paper, why I had to identify another journal to get the data published, accessible, and citable to other researchers of the human sperm physiology field. Luckily Correspondence articles at F1000 Research were suitable for exactly this.

Of note, I believe that I discussed several issues raised in the 2018 PNAS exchange quite thoroughly in the 2020 Molecular Human Reproduction paper, e.g., the claim that $\mathrm{Ca}^{2+}$ signals must be recorded specifically from the principal piece of the flagellum only to be able to observe an inhibitory effect on CatSper in human sperm cells, which did not agree with my observations using the other CatSper-inhibitor RU1968. However, if other reviewers also suggest this, we can of course update our Correspondence to discuss the issues raised in the 2018 PNAS exchange more thoroughly.

I fully agree with the reviewer that this Correspondence may not solve this controversy. However, in a recent review article "Natural Products with Potential for Nonhormonal Male Contraception" (J. Nat. Prod. 2021, 84, 2762-2774), they also discuss the controversy regarding the action of Lupeol and Pristimerin on human CatSper, and state the following: "These contradictory findings suggest a need for further studies to confirm the action or lack of action of the plant triterpenoids on the CatSper channel. The conflicting results also demonstrate the difficulties in reproducibility of results, which is often a barrier to studies of natural compounds in general.".

I believe that we are following up on this exact suggestion with this Correspondence and I hope it will be helpful for solving the issue and reaching consensus in the human sperm physiology field at some point.

In the end, my hope is that other researchers reading our Correspondence will obtain Lupeol and Pristimerin themselves and test these compounds for effects on CatSper in human sperm cells in their own lab and hopefully at some point publish their findings like we have done here. This will be the only way to solve this controversy in my opinion.

Competing Interests: No competing interests were disclosed.

Author Response 05 Jul 2022

\section{Anders Rehfeld}

As the other reviewers also suggest that we discuss the issues raised in the 2018 PNAS exchange, we have now update our Correspondence manuscript to address these issues more thoroughly.

Competing Interests: No competing interests were disclosed. 
The benefits of publishing with F1000Research:

- Your article is published within days, with no editorial bias

- You can publish traditional articles, null/negative results, case reports, data notes and more

- The peer review process is transparent and collaborative

- Your article is indexed in PubMed after passing peer review

- Dedicated customer support at every stage

For pre-submission enquiries, contact research@f1000.com 